\title{
Research on cloud manufacturing platform for small and medium-sized enterprises
}

\author{
Shujie $\mathrm{Ke}^{1}$, Shengkai Chen ${ }^{1}$, Mengnan Liu ${ }^{1}$, Cunzhi $\mathrm{Xu}^{1}$, Jie $\mathrm{Bai}^{1}$, and Shuiliang Fang ${ }^{1, *}$ \\ ${ }^{1}$ College of Mechanical Engineering, Zhejiang University, Hangzhou, People's Republic of China
}

\begin{abstract}
Keywords: Cloud manufacturing, Modeling and standardization of manufacturing services, Supply and demand matching, Remote manufacturing service monitoring.
\end{abstract}

\begin{abstract}
With the adjustment of China's economic structure, it is essential for manufacturing enterprises, especially SMEs, to improve their core competitiveness. Based on the service mode of Cloud manufacturing, this paper designs and develops a cloud manufacturing platform for SMEs. Firstly, according to the characteristics of cloud manufacturing, the overall framework of the platform is determined, and the corresponding function tree is refined. Then, the key technologies are studied, including the modeling and standardization of manufacturing services, the matching of task, the execution, the online monitoring of service, and the evaluation of post-service. The functions of service access, service matching, outsourcing task management, remote monitoring, and evaluation of tasks are realized. Finally, the platform is applied in the mold manufacturing industry, and an example analysis is carried out to verify the effectiveness of the proposed platform.
\end{abstract}

\section{Introduction}

Small and medium-sized enterprises (SMEs) are the main body of the market economy, which are the basic forces to promote national economy development and social stability. There are about 42 million SMEs in China, accounting for more than $99.8 \%$ of the total number of enterprises in the country, and $60 \%, 40 \%$ and $60 \%$ of the total industrial output value, profits and taxes, and exports, respectively [1]. Many SMEs lack the ability of product innovation, and the weak informatization base hinders the improvement of SMEs' industrial cluster management level. SMEs are in urgent need for enhancing their ability of optimizing the allocation of resources and building a new manufacturing mode to activate the diversified resources. Cloud manufacturing is a new manufacturing mode emerging in recent years, which meets the requirements above.

Li et al. [2] argued that cloud manufacturing is a new mode of networked manufacturing that manages manufacturing resources and provides services for enterprise users through a cloud manufacturing platform. Compared with the traditional networked manufacturing

\footnotetext{
*Corresponding author: me fangsl@zju.edu.cn
} 
technologies, cloud manufacturing has the following characteristics [2]: a reasonable and efficient operation mode, the strong ability of resource pooling and knowledge pooling, the personalized and socialized manufacturing capability, and the safe operation environment of manufacturing services.

Scholars all over the world have made fruitful research achievements about cloud manufacturing. In terms of the platform's architecture, Simeone et al. [3] proposed an intelligent platform to improve resources efficiency. Aiming at realizing a continuous production chain for collective cloud manufacturing, Strljic et al. [5] developed a system architecture that considered the dynamic changes of Industry 4.0. Li et al. [6] proposed a distributed peer-to-peer network architecture based on block chain technologies to improve the security and scalability of cloud manufacturing.

As for resources' description and encapsulation, aiming to solve the problem of standardized expression and sharing of massive cloud manufacturing services, Bai et al. [7] proposed a standardized cloud service modeling method for product life cycle based on ontology and standard service list structure. Li et al. [8] proposed a metadata-based ontology modeling method for manufacturing resources to solve the problem of manufacturing resources' consistency description.

In terms of service selection and scheduling, Liu et al. [9] proposed a decomposition algorithm for tasks based on sequential task decomposition. Resource matching problem in the decomposition process are considered. Helo et al. [10] introduced a cloud-based production scheduling system for sheet metal manufacturing, and discussed the requirements of "scheduling as a service".

As for the cloud manufacturing platform that in using, the CASI cloud platform and MFG.com are representative at home and abroad. The CASI cloud platform [11] is an industrial Internet platform, which provides businesses and individuals with services such as intelligent research and development, intelligent production, and so on. MFG.com [12] is the world's largest manufacturing online market. It provides resource access services for manufacturing service providers and matches the required services for users to seek manufacturing services on the platform.

Most of the studies above focus on the cloud platform's mechanism, but few attentions are paid to the cloud platform for the SMEs. Combined with the characteristics of manufacturing industry and the needs of SMEs, a cloud manufacturing platform for SMEs are proposed in this paper.

\section{The overall design of the cloud manufacturing platform}

\subsection{Architecture of the cloud manufacturing platform}

Based on the status and needs of SMEs, this paper proposes a framework of the cloud manufacturing platform according to the aforementioned background and the conceptual application model as shown in Fig. 1. 


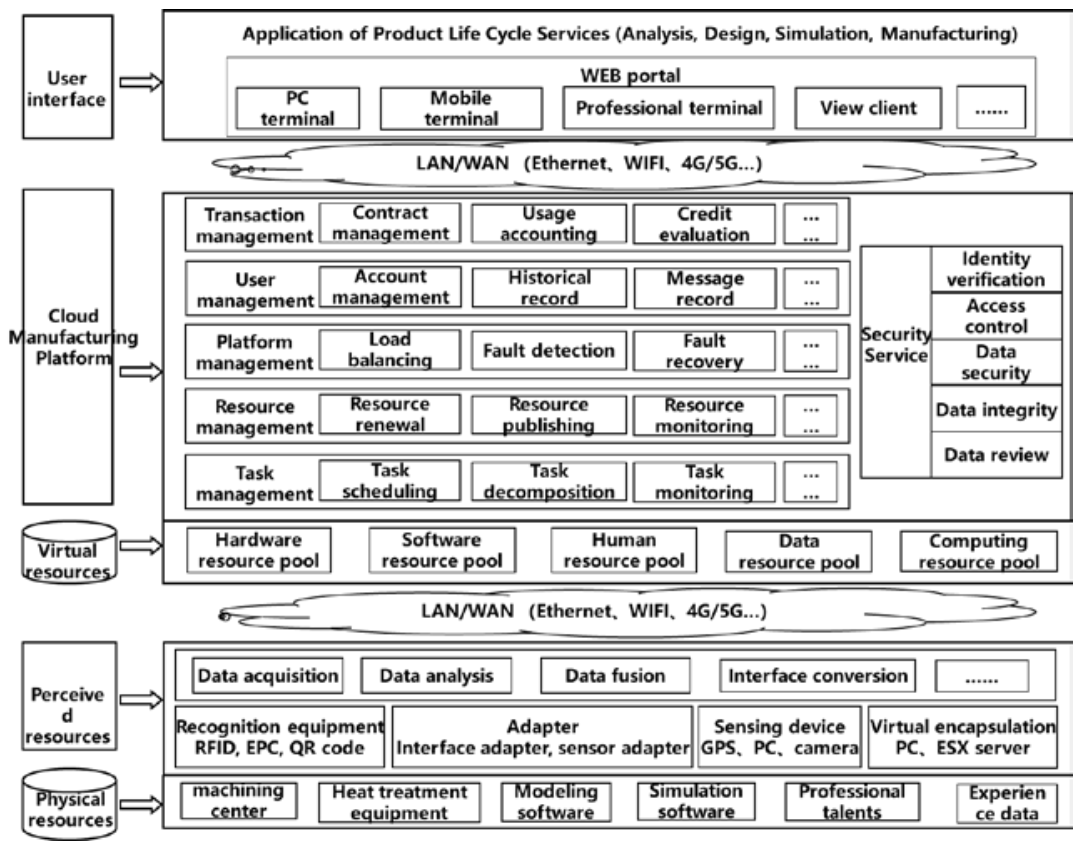

Fig. 1. The framework of the cloud manufacturing platform for SMEs.

The whole cloud manufacturing platform can be divided into five layers:

(1) Entity resources: it includes all kinds of manufacturing resources;

(2) Perception resources: it's acquired by data acquisition, analysis, and processing of real manufacturing resources through RFID, sensors, cameras, and other sensing devices;

(3) Virtual resources: it includes hardware resources, software resources, human resources, and data resources, etc.;

(4) The cloud manufacturing platform: it includes many functions, such as transaction management, user management, platform management, resource management, task management, and so on;

(5) User interfaces: it includes PC, mobile devices and all kinds of application services provided to users through interfaces by the cloud manufacturing platform.

The key operational modes of the cloud manufacturing platform are introduced in detail in the following subsections.

\subsection{Role management system}

Considering the complexity of the cloud manufacturing business, accounts are divided into two types of roles: administrators (including platform administrators, task administrators in projects, and resource/service administrators) and users (including individuals, enterprise administrators, and internal users in enterprises). Different roles have different permissions as shown in Fig. 2. 


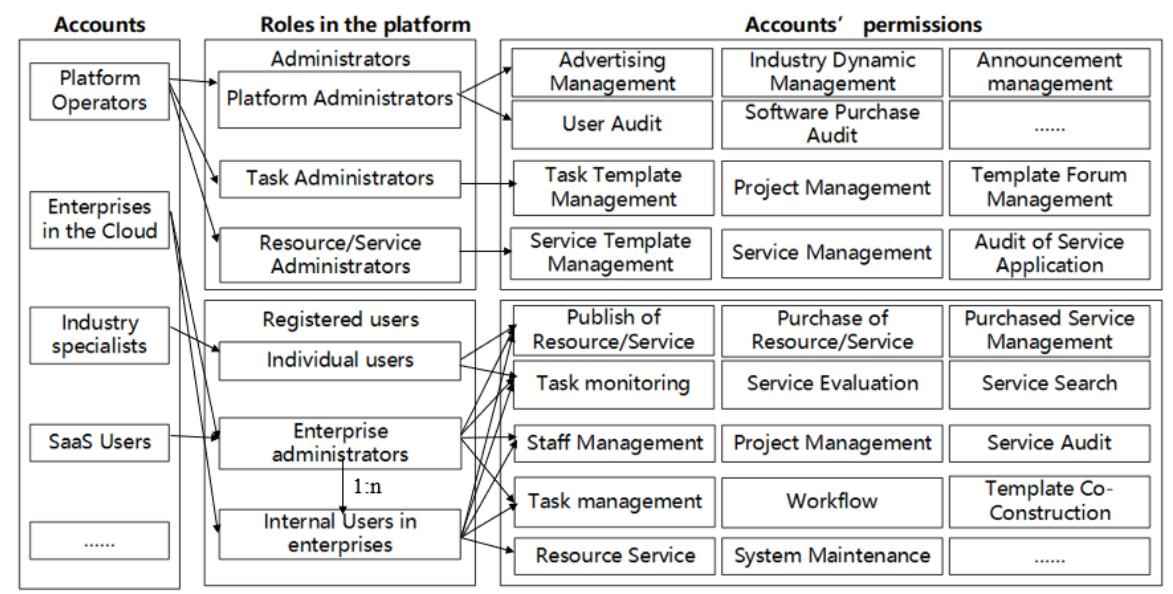

Fig. 2. The role management function.

\subsection{User service mode and the platform's profit mode}

Based on the functional architecture as shown in Fig. 1, two types of services are provided by the platform for users:

- Push service: service providers are selected by users after searching, or can be pushed to users by the platform according to the principle of minimum cost, minimum time and so on;

- One-stop service: the platform involves all steps of manufacturing. After users put forward manufacturing requirements, the platform automatically gives complete solutions according to users' needs. Users do not need to select the corresponding enterprises one by one.

Corresponding to this service mode, the platform's income mainly includes:

(1) The membership fees. Besides offering discounts on transaction fees, the platform also provides members with more value-added services, such as enterprise intelligence, data reports, etc.;

(2) The advertising fees. The platform charges advertising fee for publishing three-dimensional product models in the product parts libraries;

(3) The service fees. Such fee is charged by providing high-level technical services and consulting services for manufacturing enterprises, such as design and manufacturing consulting services.

\subsection{Resource/service operation and transaction mode}

(1) The collection and supply of resources and services on the cloud manufacturing platform:

- Centralized purchase: according to the needs of SMEs, operators on the cloud platform purchase a large number of manufacturing resources, and provide services for SMEs through service encapsulation and publishing of business capabilities;

- Decentralized utilization: through the registration of resources on the platform, the idle and affluent resources of SMEs can be dynamically absorbed, and provided for others after service encapsulation in accordance with certain transaction modes.

(2) The transaction mode of resources and services on the cloud manufacturing platform

On the platform, users trade manufacturing services mainly in two ways: 
- Direct purchase: after searching and matching, users negotiate with providers and purchase resources and services directly;

- Outsourcing services based on projects: task administrators in projects need to realize the transaction, sharing and monitoring of manufacturing services on the platform according to the steps of task decomposition, outsourcing application, negotiation, and so on. This kind of transaction is more complex, but it can also achieve stronger functions.

(3) The usage mode of resources and services on the cloud manufacturing platform:

After finding required services based on their description information and completing all the business steps, the services should be invoked by users to execute the tasks. Different types of services can be invoked in different ways. Generally, they can be divided into the following types:

- On-line invocation: it's the services provided by the virtualization, such as software, data, experts and so on, which can be invoke through network information exchange between providers and demanders. This kind of services include: 1) enterprise management software services provided by CRM and ERP, 2) analysis and simulation software such as SolidWorks, 3) online consultation or remote operation services provided by experts, etc.

- Semi-online invocation: it is suitable for services that need the combination of online information exchange and offline execution, such as experiment services. Demanders can upload related documents such as the experiment's requirements to the platform in the form of the task's attachments. After determining the services, providers will download related materials online, execute tasks offline, and then return the results to the demanders.

- Off-line invocation: The platform isn't involved in specific execution of tasks, such as common batch processing services. In this case, the platform is only used as a middleware for publishing, searching, and trading services' information, and the services are carried out offline.

\section{The function design of the cloud manufacturing platform}

Based on the overall framework and operational service model mentioned above, the function tree of the platform is refined as shown in Fig. 3. 


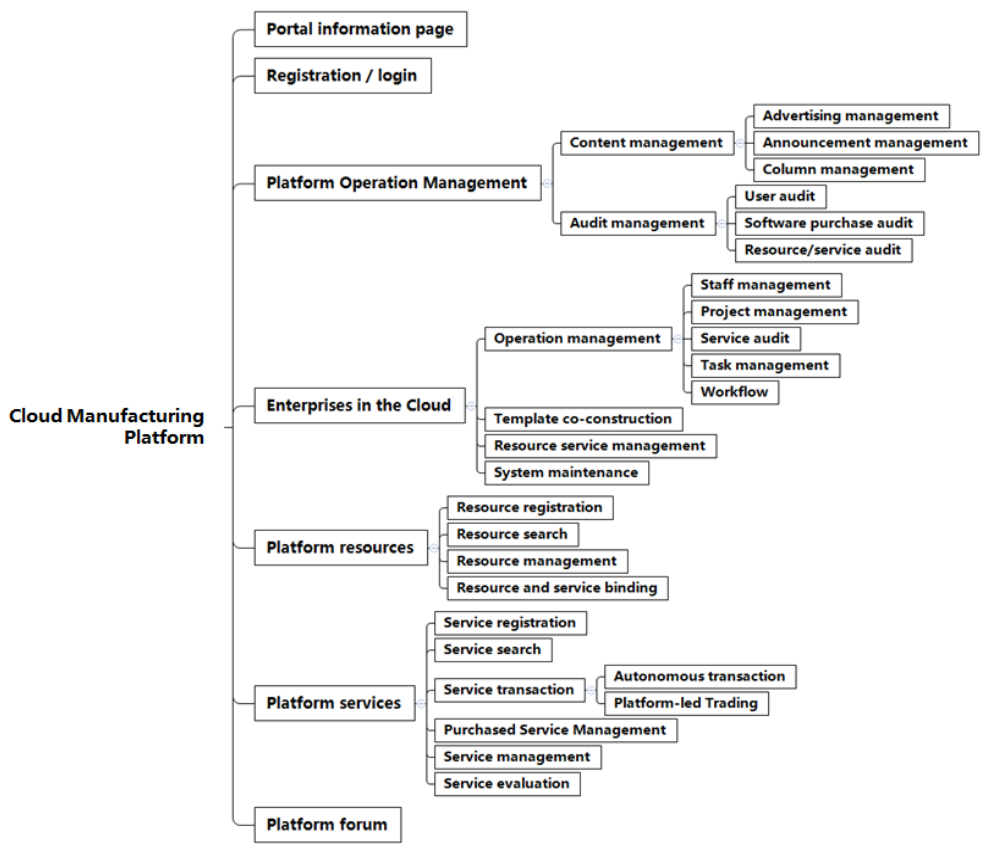

Fig. 3. Functions structure of the platform.

The main functions that orient resource/service demanders and resource/service providers on the platform are addressed in detail.

\subsection{Resource management module}

Resource management module has the following features:

1. The module provides standard registration templates for different types of services/resources, and all the relevant operations are based on these templates;

2. Users can discover services through the joint search by categories and multiple conditions;

3. The module supports users to submit purchase applications for required resources/services, and also supports users to register their own resources and services independently;

4. The module integrates CARA (Cloud Accessing Resource Adapter) module in order to support real-time monitoring and managing the task processing for demanders;

5. The module supports users to evaluate services and generate total score of services.

\subsection{Task management module}

If users choose to outsource their tasks on the platform, they need to take advantage of the task management module. The operation flow of task management system is shown in Fig. 4. 


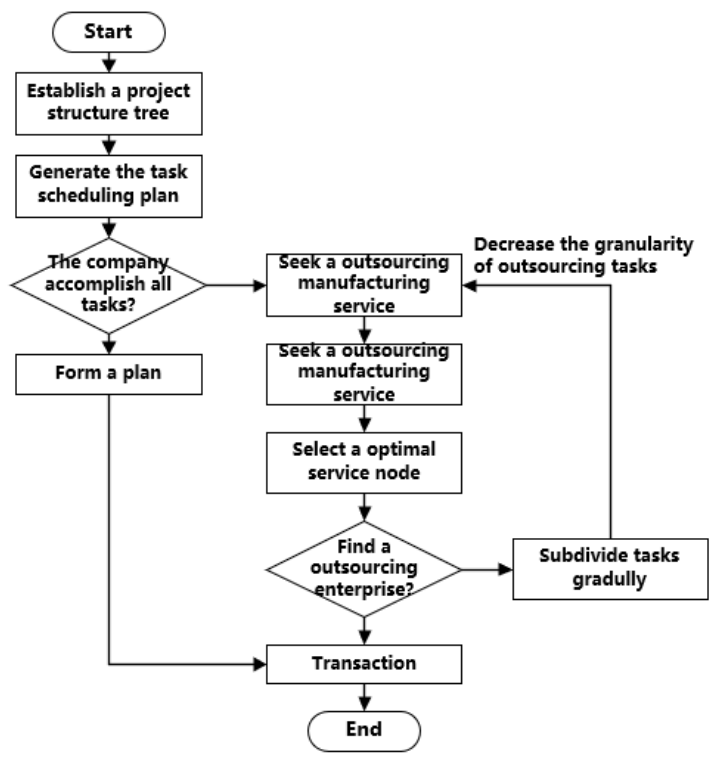

Fig. 4. Decomposition process of outsourcing tasks.

After the enterprise establishes a project structure tree and generates the project scheduling plan, the tasks, which cannot be completed and outsourced on the platform by the enterprise, will be decomposed gradually until the transaction is completed.

\section{Key technologies of the cloud manufacturing platform}

\subsection{Manufacturing services modeling and standardization technology}

Due to the diversification of manufacturing resources and services in manufacturing industry, it is difficult to create cloud manufacturing services. In order to organize and manage massive manufacturing resources and services effectively in the cloud manufacturing environment, it is necessary to model and describe cloud manufacturing services in a reasonable way.

In this paper, cloud manufacturing services are classified into the following three categories:

- Atomic services: this type of manufacturing service is the foundation of the cloud manufacturing platform, which is a kind of indivisible cloud manufacturing services formed by a single resource or a combination of resources;

- $\quad$ Enterprise-level integrated manufacturing services: it includes cloud manufacturing services encapsulated by enterprises in the form of atomic services' sets;

- Platform-level integrated manufacturing services: it refers to integrated manufacturing services encapsulated by combining the manufacturing capabilities of multiple enterprises due to the requirements of tasks in projects.

In order to enable different enterprises in industry to model and describe cloud manufacturing services in a standardized, convenient, and fast manner, and achieve services' standardized modeling and systematic management on the platform, a modeling method of standard manufacturing service is proposed in this paper after referring to BOM technology, 
and a product-life-cycle-oriented tree about the Bill of Standard Manufacturing Services (BOSS) is also built by the following steps.

Step 1: Define the atomic services and determine the set of services in the atomic layer;

Step 2: According to the set of atomic services defined by Step 1, a model for allocating manufacturing resources/services is established to determine the set of atomic services in BOSS tree;

Step 3: According to BOM and industry standards, a BOSS tree is constructed by the platform;

Step 4: Referring to the BOSS tree, the business capabilities and tasks are encapsulated as cloud manufacturing services by enterprises on demand;

Step 5: Enterprises encapsulate manufacturing services' information to discover frequent item sets according to association rules and determine standard integrated manufacturing service sets. The correlation between atomic manufacturing services is explored from the mass of manufacturing service information encapsulated by enterprises' registration. The atomic service set of platform standard integrated manufacturing services is built according to the correlation calculation, and then the set of standard integration services in various levels is determined and encapsulated.

Step 6: Combining with the set of services, a hierarchical BOSS is constructed based on the polychromatic set theory. The logical relationship among the standard integrated manufacturing services is determined by the logical operation, and the BOSS tree is constructed finally.

\subsection{Task matching and execution technology}

Task matching and execution technology is important in task management module. According to the conditions of tasks' execution, there are three forms to match tasks with resources/services.

- The task is carried out independently by the enterprises without matching.

- The task is matched with resources. The enterprises have production capacity, but lack part of resources.

- The task is matched with services. The enterprises lack both production capacity and production resources.

After determining the task execution mode, next step is to retrieve the optimal resource/service node on the platform. Generally speaking, the retrieval process is divided into two steps.

- The first step is to match the information of the outsourcing task with that of the service nodes on the platform, and select a certain number of candidate service sets (CSS).

- The second step is to rank the candidate service nodes in the first step according to the TQCS (time, quality, cost, and service) constraints and other information of the outsource task. The top-ranking service node that satisfies the requirements will be selected as the optimal service node.

The service retrieval process is shown in Fig. 5.

Next, the details of these two steps are described below.

(1) Preliminary matching

Obtaining CSS by preliminary matching is the first step to select the optimal service node. Enterprises select CSS by matching the information of the outsourcing tasks with that of candidate services. A preliminary matching algorithm is proposed as follows:

A. Precision matching: The attributes of the services to be matched must satisfy requirements for values of the tasks' attributes. The values of tasks' attributes always require 
certain values or a smaller range of value, i.e. cservice $_{i}$. property $=$ tas $_{j}$. property. The number of matched nodes obtained by this rigorous method is usually small.

B. Fuzzy matching: the information of tasks has a certain range of attributes, and the attributes' values of matched services should be screened and included in this range.

C. Flexible matching: the attributes will be arranged in a certain order of priority. According to the priority order, attributes of the high priority will be satisfied first in the matching process.

Enterprises on the platform generally choose fuzzy matching and flexible matching. Through similarity calculation, semantic matching, and so on, the matched nodes are presented form a candidate service set, CSS = cservice $_{1}$, cservice $_{2}$, cservice $_{3}, \ldots$, cservice $\left._{n}\right\}$, where cservice $_{i}$ denotes the first service node and $\mathrm{N}$ is the number of service nodes in the candidate service set.

According to the number of service nodes $\mathrm{n}$ contained in the candidate service set, it can be processed in the following situations:

(1) $\mathrm{n}=0$, that is, the platform does not get a suitable service node. Under this circumstance, the matching requirements of tasks' attributes should be adjusted by expanding the range of attributes' values and reducing the priority of an attribute, and then re-matching.

(2) $\mathrm{n}=1$, that is, the platform gets a service node that meets the requirements of tasks' attributes. Enterprises will evaluate the service node, and the matching process is completed if the evaluation is passed; if not, this service node will be deleted in CSS, and then match again as (1).

(3) $1<\mathrm{n} \leqslant$ Max, where Max is a constant ranging from 10 to 15 . The optimal service node needs to be selected by the ranking algorithm introduced in the following subsection.

(4) $n>$ Max. Under this circumstance, the number of matched service nodes is large, so the requirements of tasks' attributes need to be adjusted, and then re-matching.

(2) Ranking algorithm.

After preliminary matching, if there are several service nodes in the candidate service set, it is necessary to select the optimal service node by selecting the evaluation indexes and determining the indexes' weight according to the constraints of TQCS.

According to the matching results of tasks and services, a selection model based on AHP is established to select the optimal service node. Its detailed structure is shown in Fig. 6.

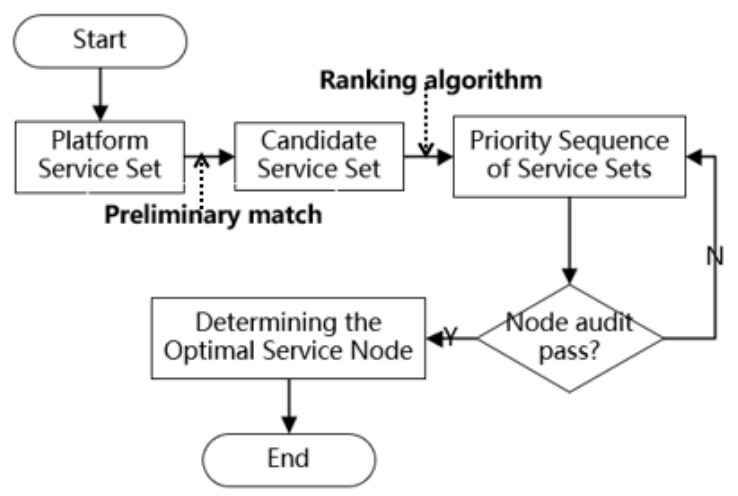

Fig. 5. Flow chart of retrieving the optimal service node. 


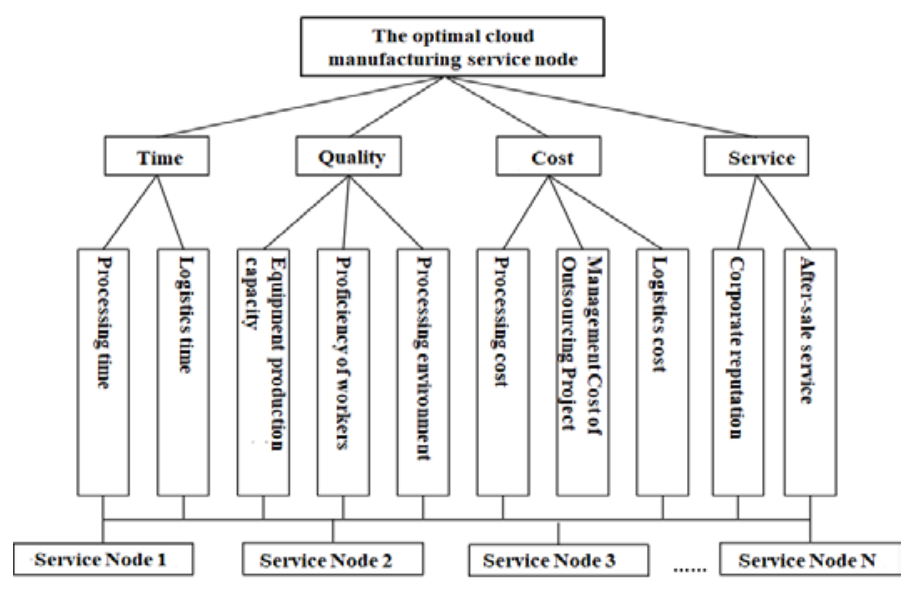

Fig. 6. AHP model for selecting the optimal service node.

After establishing the selection model, it is necessary to determine the indexes' weight coefficients in the model. The expert will evaluate this model, which assigns weight coefficients from top to bottom by task publishing enterprises, is relatively complex and reflects the subjective will of enterprises. However, the entropy weight coefficient method derives weight coefficients from parameter information of candidate service nodes from bottom to top, which is easy to calculate and relatively objective. Referring to the expert evaluation model and entropy weight coefficient method based on Delphi method, the final coefficients of indexes' weight are obtained after synthesizing the results obtained by the two methods. The optimal service node selection method is shown in Fig. 7.

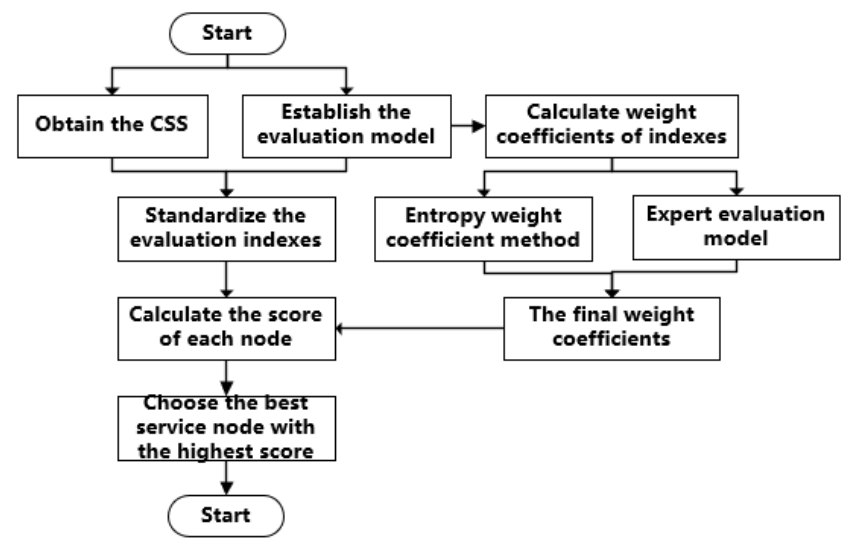

Fig. 7. Flow chart of the optimal service node selection.

\subsection{Online task monitoring technology}

It is necessary to monitor the implementation of tasks, to record their progress, and then manage the workflow of enterprises and adjust the execution plan of related tasks according to feedback. Local tasks are monitored by the enterprises, while outsourcing tasks are monitored by the enterprises and service providers through the monitoring interfaces on the cloud manufacturing platform. 
The main steps of monitoring outsourcing tasks are as follows: after task outsourcing, the platform provides a task monitoring interface. The monitoring data will be stored on the cloud manufacturing platform database as the task information archive of enterprises.

There are 5 states of outsourcing tasks: wait for execution, in execution, accomplished, suspend, and termination. The transition relationships between various states are shown as Fig. 8.

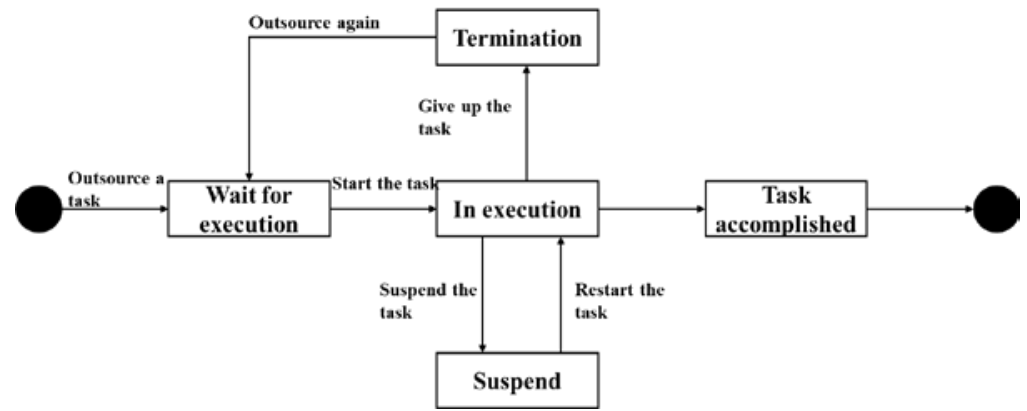

Fig. 8. Flow diagram of outsourcing tasks' status.

\subsection{Service evaluation technology}

A service evaluation scheme is presented based on user evaluation to evaluate the quality of manufacturing services. That is, the demander who publishes the task will be able to score and evaluate the service after a provider completes its task. The evaluation score reflects the quality of the service, and it will be reflected in the service's information, so as to facilitate others for reference.

The evaluation index system of manufacturing services is established based on AHP as Fig.9.

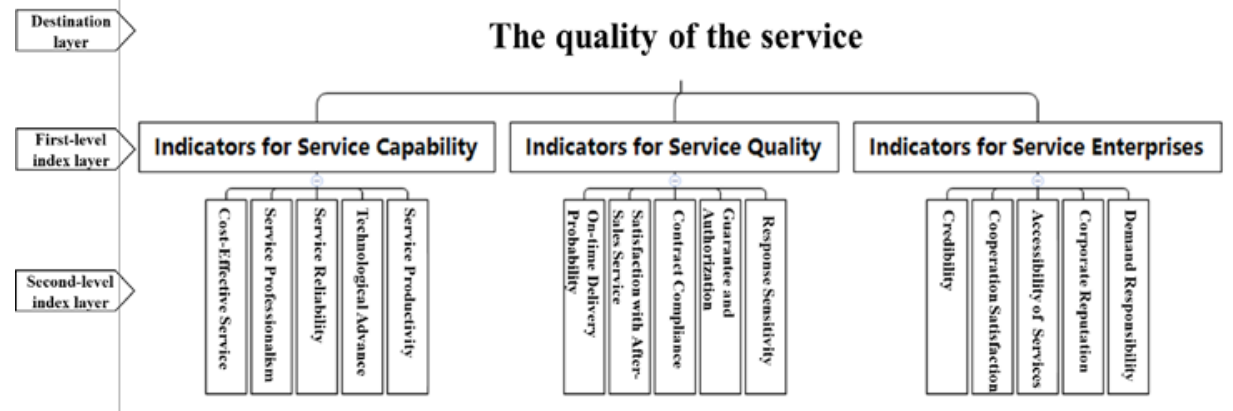

Fig. 9. The evaluation index system of cloud manufacturing services.

The evaluation index system is divided into three layers. The destination layer is the final evaluation value of the quality with manufacturing services. The second and third layers are the detailed decomposition of the destination layer.

\section{A Case study of the cloud manufacturing application}

In most industrial products, $60 \% \sim 80 \%$ of parts depend on mold forming. Fig. 10 is a simplified BOSS tree of the mold industry. 


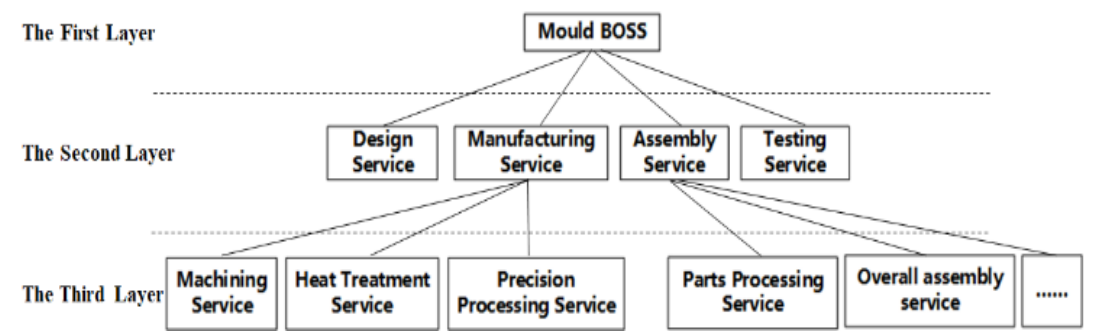

Fig. 10. The simplified BOSS tree of the mold industry.

In China, there are many SMEs of mold industry lacking international market competitiveness. Besides, most of them have long development cycle, low quality, high manufacturing costs, etc.

The proposed platform in this paper solves the problems in the following ways:

(1) The platform integrates and optimizes the resources of enterprises to realize knowledge sharing and management, logistics systematization, and operation process optimization.

(2) The platform absorbs all kinds of manufacturing resources and capabilities, and transforms them into services that are available everywhere. Besides, intermediary services including consultation, talent introduction, and so on, are provided to run through the whole production process.

After defining a project, the enterprise can assign a project manager and define the workflow in the task management module. Then, tasks at all levels will be created as Fig. 11.For tasks that need to be outsourced, the project manager publishes tasks and search for appropriate services. The specific process is shown in Fig. 12.

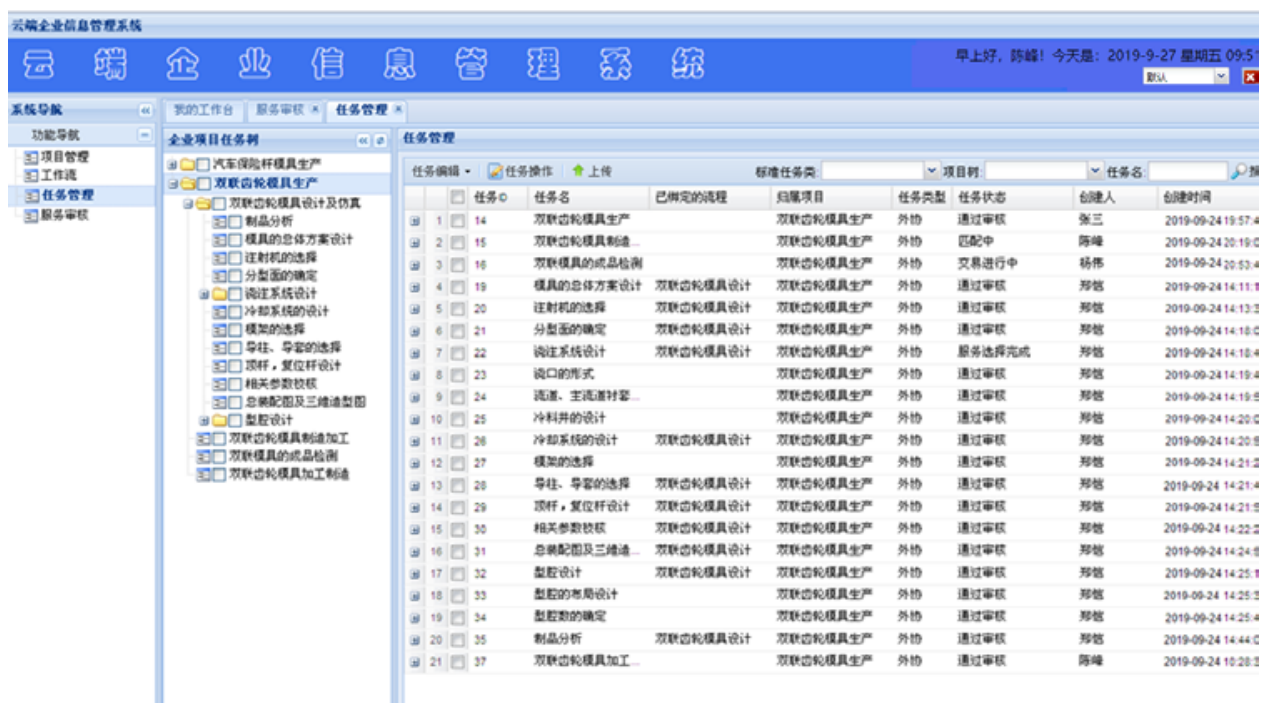

Fig. 11. Create the project's tasks. 


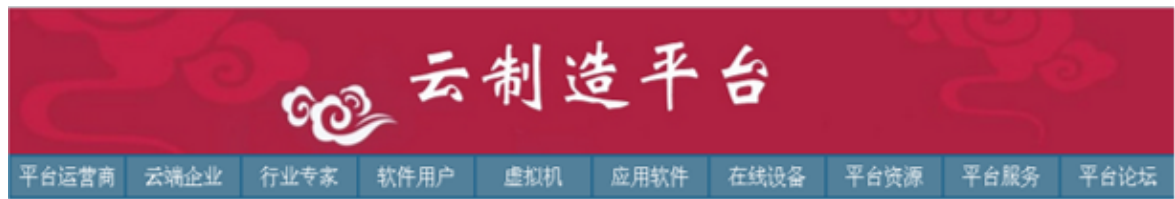

当前位置: 首页 $>$ 平台服务

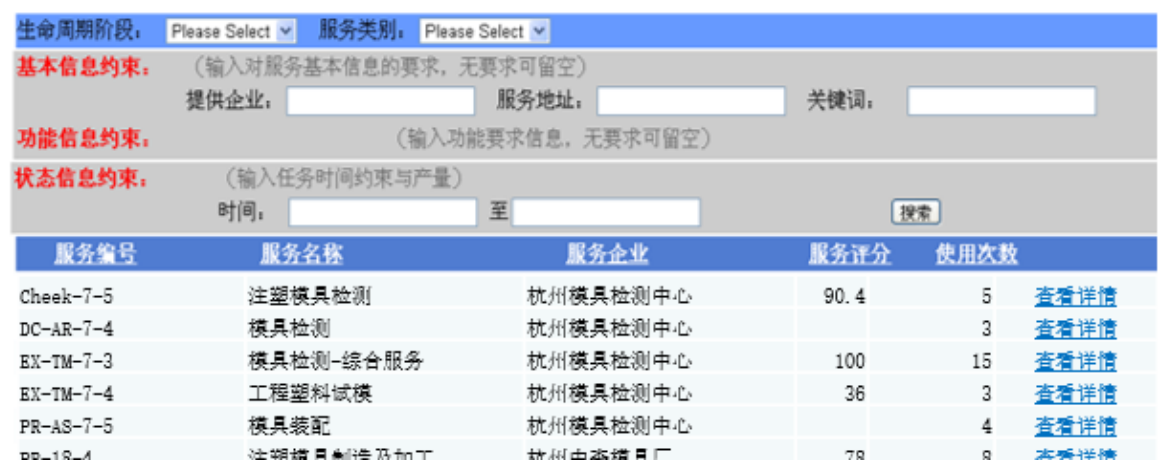

Fig. 12. Searching for services on the platform.

After finding the right service, the demander and the provider negotiate and trade. (Fig. 13). In the process of task execution, the platform provides the monitoring service for the demander (Fig. 14). In the whole process, with the help of the platform, the supplier of services improves the utilization efficiency of its resources, and the demander gets the required services conveniently.
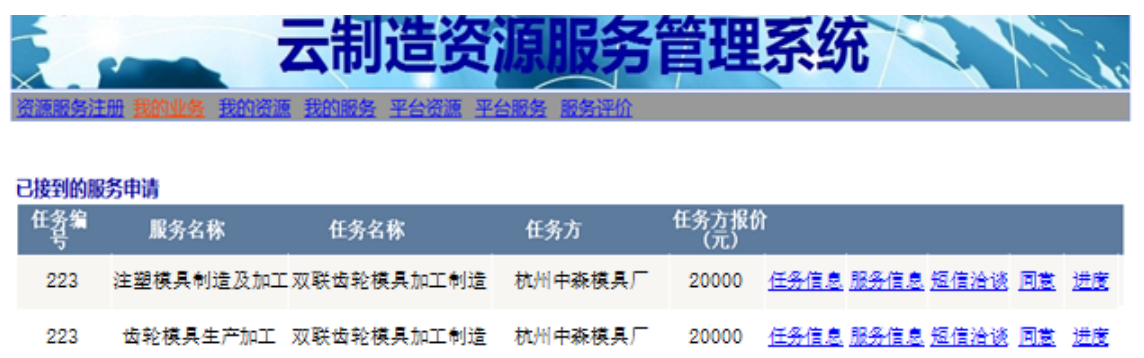

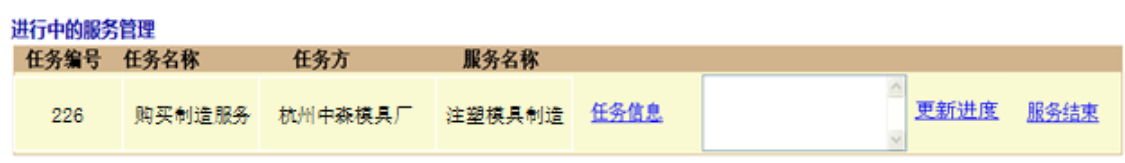

\begin{tabular}{|c|c|c|c|c|c|c|c|c|c|c|}
\hline $\begin{array}{c}\text { 发祭 } \\
\frac{\text { 号 }}{2}\end{array}$ & 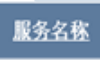 & 成务方 & & 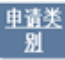 & 报佮 & 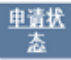 & 車䄇时间 & 交易时间 & 完虚时间 & $\begin{array}{l}\text { 操 } \\
\text { 作 }\end{array}$ \\
\hline $\mathrm{DA}-3-1$ & 市场谓咞 & 云炤多平台 & 盗看 & 购买 & 20000 & 已完成 & $\begin{array}{l}2019 / 7 / 15 \\
15: 34: 26\end{array}$ & $\begin{array}{l}2019 / 7 / 15 \\
17: 01: 31\end{array}$ & $\begin{array}{l}2019 / 7 / 10 \\
09: 36: 43\end{array}$ & 进度 \\
\hline $\begin{array}{c}\text { PR-18- } \\
4\end{array}$ & $\begin{array}{c}\text { 望棋具敷逜 } \\
\text { 加工 }\end{array}$ & 机州中数槙具 & 管登 & 购买 & 50000 & 成交 & $\begin{array}{l}2019 / 9 / 23 \\
21: 54: 13\end{array}$ & $\begin{array}{l}2019 / 9 / 23 \\
22: 43: 16\end{array}$ & & 进度 \\
\hline
\end{tabular}

Fig. 13. The transaction process. 


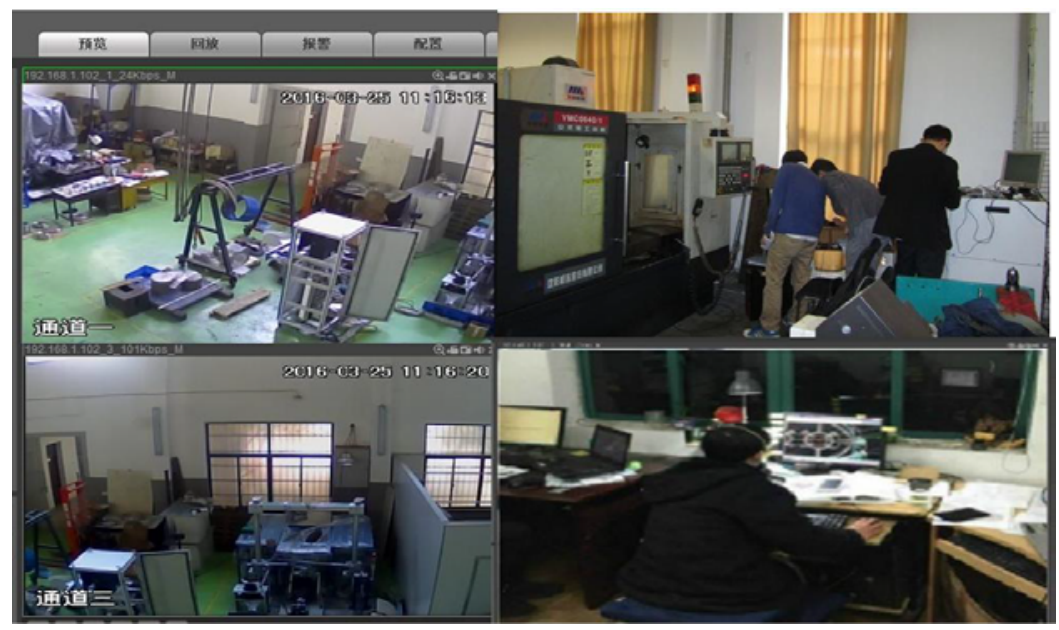

Fig. 14. On-site monitoring of remote services

\section{Conclusion}

In order to enhance the competitiveness of SMEs, the overall architecture of the cloud manufacturing platform is designed firstly. Then, several key technologies are studied, which include the modeling and standardization of manufacturing services, the matching and execution of task and so on. Finally, this paper carries out exploratory application and a case analysis in the mold manufacturing industry, which proves the effectiveness of the proposed cloud manufacturing platform.

This research is financially supported by the National Natural Science Foundation of China (71571161) and the Science Fund for Creative Research Groups of National Natural Science Foundation of China (No. 51821093).

This paper is based on the works of the author's research group for nearly ten years. Thanks to the work of the graduated students Si-yao Cheng, Xiang Fu, Shuang-sheng Luo, Sen Wang, Qing Xu, Hao-ke Peng, Fei Sheng, etc.

\section{References}

1. H. Zeng, S. Huang, Research on Innovation of Financing Model of SMEs Based on Industrial Cluster, Enterprise Economy, 12 (2012) 86-88.

2. B. Li, Z. Lin and S. Wang, Cloud manufacturing: a new service-oriented networked manufacturing model, Computer Integrated Manufacturing Systems, 16 (2010) 1-7.

3. C. Li, Y. Shang and C. Hu. Research of Structure and Key Technologies for Cloud Manufacturing, Modular Machine Tool \& Automatic Manufacturing Technique, 7 (2011) 104-107.

4. A. Simeone, A. Caggiano, L. Boun and B. Deng, Intelligent cloud manufacturing platform for efficient resource sharing in smart manufacturing networks, Procedia CIRP, 79 (2018) 233-238.

5. M. M. Strljic, D. Brovkina, T. Korb, O. Riedel and A. Lechler, Platform architecture concept for the composition of collective cloud manufacturing, 25th International Conference on Mechatronics and Machine Vision in Practice (M2VIP), 2018. 
6. Z. Li, A.V. Barenji and G. Q. Huang, Toward a blockchain cloud manufacturing system as a peer to peer distributed network platform, Robotics and Computer-Integrated Manufacturing, 54 (2018) 133-144.

7. J. Bai, S. Fang, H. Peng and R. Tang, BOSS modeling method for cloud manufacturing services normalization, Computer Integrated Manufacturing Systems, 23 (2017) 202-213.

8. X. Li, P. Zhuang and C. Yin, A metadata based manufacturing resource ontology modeling in cloud manufacturing systems, Journal of Ambient Intelligence and Humanized Computing, 10 (2019) 1039-1047.

9. M. Liu, Q. Wang and L. Lin, Cloud Manufacturing Task Decomposition Method Based on HTN, China Mechanical Engineering, 28 (2017) 924-930.

10. P. Helo, D. Phuong and Y. Hao, Cloud manufacturing - Scheduling as a service for sheet metal manufacturing, Computers \& Operations Research, 110 (2019) 208-219.

11. Information on http://os.casicloud.com.

12. Information on https://www.mfg.com.

13. S. Cheng, Research on the resources and services management mode and its prototype development for cloud manufacturing, Master. dissertation, Zhejiang Univ., Hangzhou, 2013.

14. W. Fu, Several key technologies research and preliminary prototype development for cloud manufacturing, Master. dissertation, Zhejiang Univ., Hangzhou, 2012. 